\title{
Fractal Parallel Stacked Inductor using Modified Hilbert Space Filling Curve for RFIC's
}

\author{
P. Akhendra Kumar \\ Research Scholar \\ Dept of ECE \\ National Institute of Technology,Warangal
}

\author{
N. Bheema Rao \\ Associate Professor \\ Dept of ECE \\ National Institute of Technology,Warangal
}

\begin{abstract}
High-Quality factor miniaturized inductors are prerequisites of RFIC Applications. This paper presents a novel fractal stacked inductors using modified Hilbert space filling curve. The parallel stacked inductor with differential excitation improves the $\mathrm{Q}$ factor values. The results show more than $50 \%$ improvement in $\mathrm{Q}$ over conventional fractal inductors maintaining same inductance value with the same occupying area.
\end{abstract}

\section{General Terms}

Modified Hilbert space filling curve, Radio Frequency IC's

\section{Keywords}

High Frequency Structural Simulator (HFSS),Inductance value(L), Modified fractal structure, Quality factor(Q)

\section{INTRODUCTION}

The on-chip passive inductor is a necessary and crucial element to most RF Integrated circuits [1]-|2]. High performance and cost effective RF circuits are achieved by the use of these passive inductors.

At lower frequencies, bond wire inductors are preferred as it offers high inductance, but at higher frequency requirement of inductance is in the order of $\mathrm{nH}$ such lower inductance values can't be achieved with bond wires hence on-chip inductor are preferred. High inductance density with higher Q values used at higher frequencies are the major challenging aspects of inductor modeling. Various on-chip inductors are proposed in the literature such as planar inductors, Planar inductors with pattern ground shield, Symmetric inductors, series stacked inductors and pyramidal up down structures.

Planar inductors [3] suffer from low Q factor, Low Q-factor is due to the resistive-capacitive losses on the silicon substrate, and also area constraints limit the inductance value. Pattern ground shield [4] is used to improve the Q factor in the cost of improved fabrication cost. Symmetric inductors [5]-[6] excited by differential inputs yields higher $Q$ values due to the presence of a shorter underpass; However single layer symmetric inductors occupy the larger on-chip area to achieve higher inductance values.

Series stacked inductors $[7]-[8]$ produces large inductance values with the lesser on-chip area, But this inductor suffers from lower Q factor and SRF due to the large capacitance between the adjacent layers. Interlayer capacitance reduced by adopting up and down series windings [9]-[10].thereby increase in Q factor and self-resonant frequency. Inductor designed using fractal geometry were highly area efficient [11]-[12]. An intuitive study of mathematically defined fractal space filling inductors was carried out in [13]-[14]. The unique property of space filling curves significantly increases the inductance value within the limited area. Due to the limitation of CMOS technology the construction of ideal fractal inductors cannot be built. In this present study, Convention Hilbert space filling curve is modified to re-

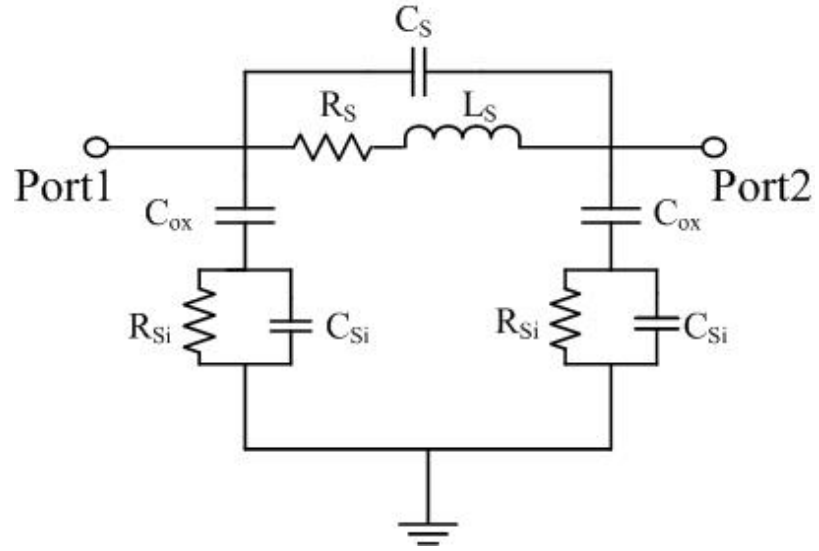

Fig. 1. Physical model of an onchip inductor

duce the lateral flux intern improves the Quality factor as well as self-resonant frequency. Further parallel stacks with differential excitation to the modified structure improves the Quality factor massively.

The organization of the paper is as follows, Construction of fractal curves is explained in section II

The basic structure of modified Hilbert space filling curve is explained in section III. Section IV reports proposed parallel stack fractal inductor. The details of the simulation results are shown in Section V, followed by concluding the summary in Section VI.

\section{DESIGN CONSIDERATIONS}

The physical model of on-chip inductor on silicon is shown in fig 1 . From the figure series branch consists of fractal curve inductance $\left(L_{s}\right)$, Series resistance $\left(R_{s}\right)$ represents the eddy current losses and the energy losses due to the skin effect in the metal tracks. Series resistance $\left(C_{s}\right)$ represents the overlap capacitance between the adjacent layers. The parasitics in shunt branch are modeled by $C_{o x},\left(C_{s}\right)$ and $\left(R_{s i}\right.$ (, where $\left(C_{o x}\right)$ represents the capacitance between oxide and substrate, $C_{s i}$ is the silicon substrate capacitance. $\left(R_{s i}\right)$ represents the ohmic losses signifies the energy dissipation in the substrate.

\subsection{Quality Factor}

The Quality factor of an inductor is the amount of energy stored in the amount of energy dissipated in one complete cycle and is given in equation 1

$$
Q=2 \pi \frac{\text { Energy stored }}{\text { Energy dissipated in one oscilation cycle }}
$$

For an inductor always energy stored in the form of magnetic field. There may be some energy stored in the form of the electric field due to some inevitable parasitic capacitance in a practical inductor. 


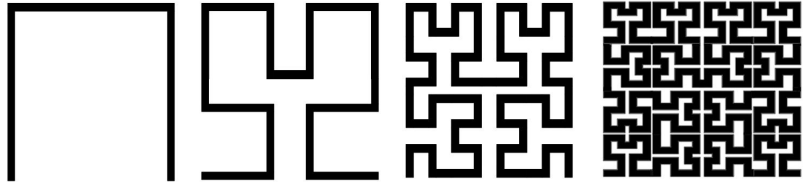

Fig. 2. Construction of Conventional Hilbert curve

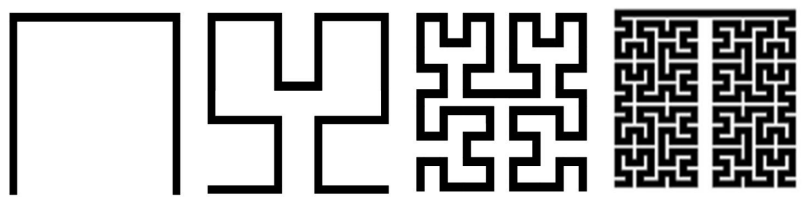

Fig. 3. Construction of Modified hilbert curve

$$
Q=2 \pi \frac{\text { Peak magnetic energy }- \text { Peak electrical energy }}{\text { Energy dissipated in one oscillation cycle }}
$$

From the above equation $2 \mathrm{Q}$ vanishes to zero at both magnetic energy and electrical energy are equal. The frequency at which $\mathrm{Q}$ is zero known as Self Resonant frequency.

\subsection{Self Resonant frequency}

The resonance frequency can be viewed as the frequency at which the peak magnetic and electric energies are equal. Thus, SRF can be modeled by a lumped RLC tank with equation 3

$$
F_{S R}=\frac{1}{\sqrt{2 \pi L_{e q} C_{e q}}}
$$

where $L_{e q}$ and $C_{e q}$ are the equivalent inductance and capacitance of the structure, respectively.

\section{MODIFIED HILBERT FRACTAL INDUCTOR}

Space filling curve is a continuous curve that passes through every point of a bounded region Most space filling curves exhibit degree of self-similarity. Many space filling curves are suggested over the years such Hilbert, Peano, etc., fig 2 shows the first four stages of conventional Hilbert space filling curve. Inductors designed using conventional Hilbert space filling curve suffers from low Q factor at higher iterations. To improve the Quality factor of an inductor Modified Hilbert space filling curve is derived from the conventional Hilbert space filling curve and is shown in fig 3 . From the fig 3 , the first three stages of Modified Hilbert space filling curve is similar to conventional space filling curve. Third iteration designed to provide some hallow space and also designed to be symmetric both geometrically and electrically at the axis of symmetry.

\section{SINGLE ENDED AND DIFFERENTIAL EXCITATION}

The Modified hilbert fractal inductor excited using both single ended as well differential ended excitation. In single ended excitation output port is terminated to ground where as in differential ended excitation, two input are in anti phase to form an virtual ground in the mid way of symmetric circuit. By using differential excitation series resistance $R_{s i}$ doubled and also parasitic capacitance's $C_{o x}$ and $C_{s i}$ halved. Because of increased series resistance and decreased capacitance almost $50 \%$ reduction in eddy current circulation in substrate. The input impedence of the inductor is calculated based on single and differential excitation

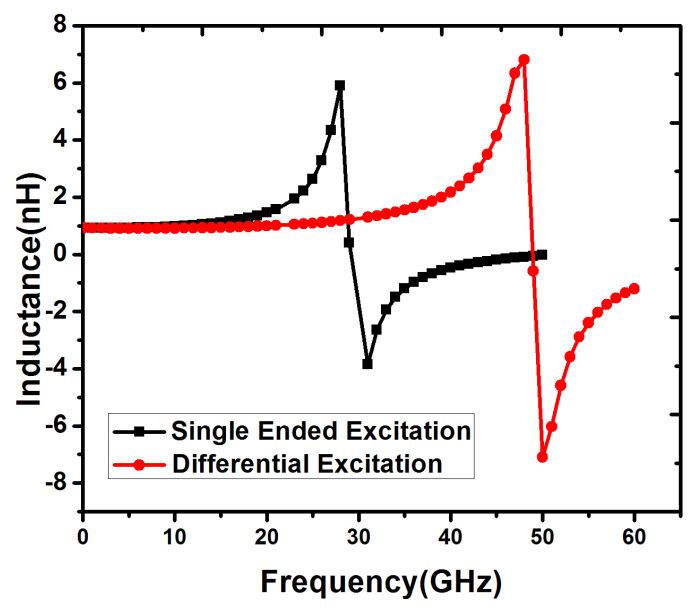

Fig. 4. Comparision Inductance plots of single ended and Differential ended excitation

are given in equation 4 and equation 5

$$
\begin{gathered}
Z_{s e}=\frac{1}{2}\left[\frac{1}{Y_{11}}+\frac{1}{Y_{22}}\right] \\
Z_{D e}=\left[\frac{Y_{11}+Y_{12}+Y_{21}+Y_{22}}{Y_{11} * Y_{22}-Y_{12} * Y_{21}}\right] \\
L_{s e}=\frac{\operatorname{Im}\left(Z_{s e}\right)}{2 * p i * f r e q} \\
L_{D e}=\frac{\operatorname{Im}\left(Z_{D e}\right)}{2 * p i * f r e q} \\
Q_{s e}=\frac{\operatorname{Im}\left(Z_{s e}\right)}{\operatorname{Re}\left(Z_{s e}\right)} \\
Q_{D e}=\frac{\operatorname{Im}\left(Z_{D e}\right)}{\operatorname{Re}\left(Z_{D e}\right)}
\end{gathered}
$$

From the input impedence, the inductance and $\mathrm{Q}$ factor are calculated using the equations. 69. The inductance and $\mathrm{Q}$ factor plots are shown in fig 4 and fig 5 for single ended and differential excitation. The effect of differential excitation is less significant at lower frequencies, where as the effect of differential excitation is more significant at higher frequencies due to the decrease in parasitic capacitance's. The simulation results shows an improvement $30 \%$ in Quality factor and $25 \%$ improvement in self resonant frequency over single ended excitation.

\section{SHREDDED PARALLEL STACKED MODIFIED FRACTAL INDUCTOR}

Fig 6 shows the 3D representation of the proposed inductor. The two metal layers of the inductor are connected by vias in parallel, thus forming a cavity in a part of metallic layers. The cavity formed between the layers improves the magnetic coupling between the layers which results almost identical inductance value with single layer fractal inductor. The improvement in $\mathrm{Q}$ factor is observed due to the minimization of skin effect. In traditional parallel inductors [15], the improvement the Q factor of the inductor the top metal is stacked with few lower metal layers through vias to minimize the overall series resistance but increases the metal substrate capacitance hence degradation in Self resonant frequency and also reduces the inductance by more than $50 \%$. 


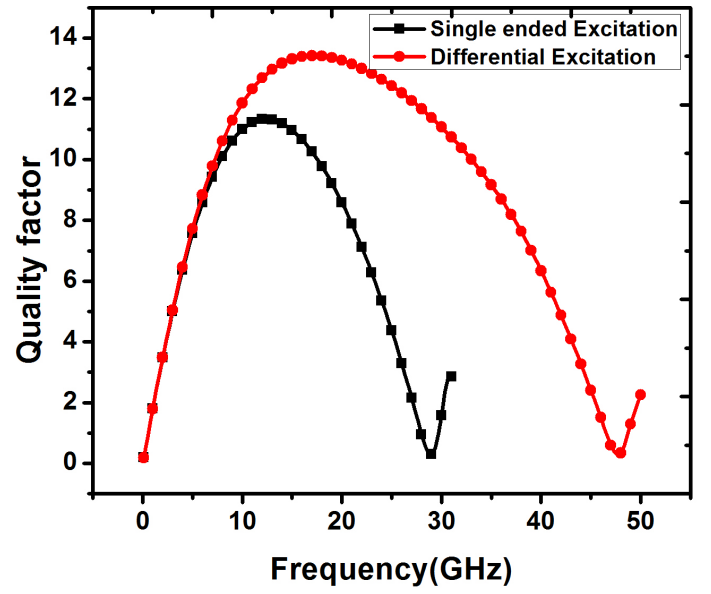

Fig. 5. Comparison $\mathrm{Q}$ factor plots of single ended and Differential ended excitation

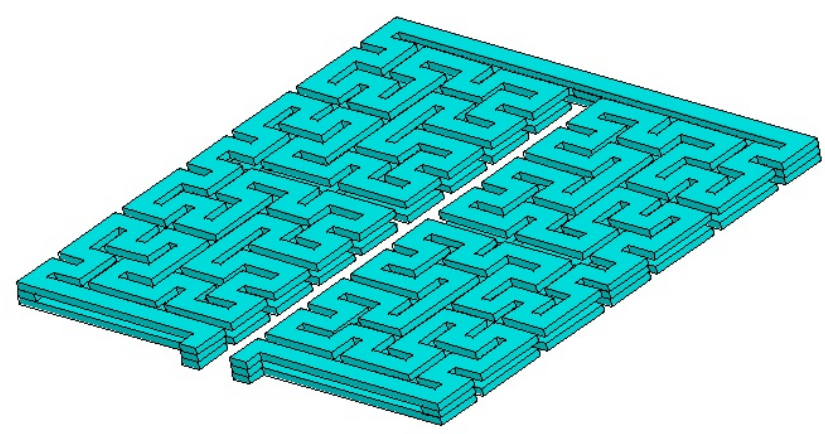

Fig. 6. 3-D representation series stack modified Hilbert inductor

\section{RESULTS AND DISCUSSION}

To quantify the advantage of the proposed architecture for parallel stacked inductors. The top metal and the bottom metal are of $3 \mu \mathrm{m}$ thick with a $2 \mu \mathrm{m}$ interlayer dielectric.

\subsection{Impact of Modified Architecture}

Two different fractal inductors are considered to validate the performance of proposed inductors. Where all these inductors designed with an effective conductive width $8 \mu \mathrm{m}$ and with an occupying area of $200 \times 200 \mu \mathrm{m}^{2}$ Fig 7 and fig 8 shows the simulated $\mathrm{L}$ and $\mathrm{Q}$ characteristics of proposed and existing standard inductors. From fig 7, It is observed that the proposed inductor acheiving highest self resonant frequency of $40 \mathrm{GHz}$ without any degradation in Inductance value. Similarly from fig 8 It is obtained that the proposed inductor acheiving highest $\mathrm{Q}$ factor of 16 which is almost $50 \%$ higher than than existing fractal reported. The Quality factor and Self resonant frequency of the existing fractal inductors are limited by resistance losses and capacitance losses. Due to the electric and axis symmetry of the modified structure and shredded parallel stacking with differential excitation improves the Quality factor and Self resonant frequency of the proposed structure.

\section{CONCLUSION}

In this study, a novel fractal stacked inductors using modified Hilbert space filling curve has been proposed. The performance

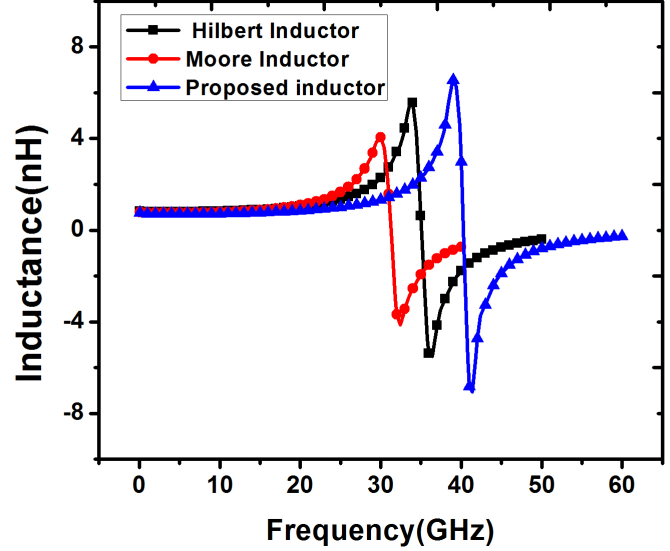

Fig. 7. Comparison of Inductance values of various fractal inductors

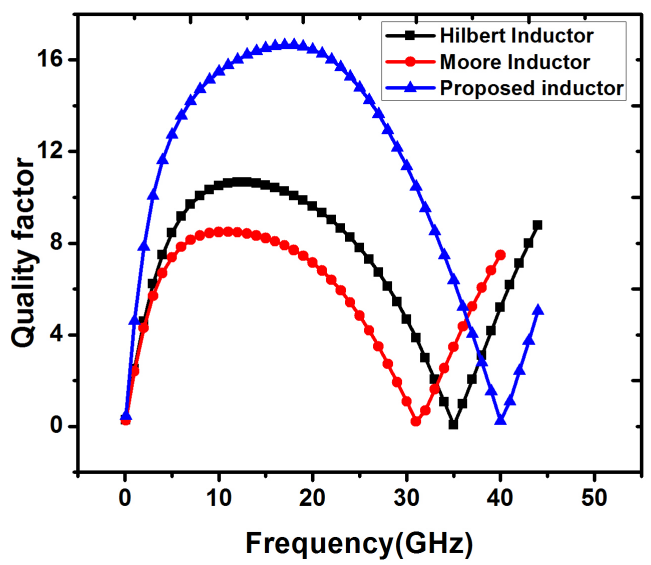

Fig. 8. Comparision of $\mathrm{Q}$ factor values of various fractal inductors

merits such as Inductance, Q factor and self resonant frequency have been studied. The results show that proposed structure achieves 50\% improvement in $\mathrm{Q}$ factor over standard fractal inductors. The proposed fractal inductor is well suited for RF front end design applications.

\section{REFERENCES}

[1] LI Baimei, UC Wang, Minglin MA, and Shengqiang Guo. An ultra-low-voltage and ultra-low-power $2.4 \mathrm{ghz}$ lna design. Radio Engineering, 18(4):527-531, 2009.

[2] J. Craninckx and M. S. J. Steyaert. A 1.8-ghz low-phasenoise cmos vco using optimized hollow spiral inductors. IEEE Journal of Solid-State Circuits, 32(5):736-744, May 1997.

[3] Cong Wang and Nam-Young Kim. Analytical optimization of high-performance and high-yield spiral inductor in integrated passive device technology. Microelectronics Journal, 43(3): 176 - 181, 2012.

[4] C. P. Yue and S. S. Wong. On-chip spiral inductors with patterned ground shields for si-based rf ic's. pages 85-86, June 1997.

[5] M. Politi, V. Minerva, and S. C. d'Oro. Multi-layer realization of symmetrical differential inductors for rf silicon 
ic's. In 2003 33rd European Microwave Conference, pages 159-162, Oct 2003.

[6] M. Danesh, J. R. Long, R. A. Hadaway, and D. L. Harame. A q-factor enhancement technique for mmic inductors. In 1998 IEEE MTT-S International Microwave Symposium Digest (Cat. No.98CH36192), volume 1, pages 183-186 vol.1, June 1998.

[7] A. Zolfaghari, A. Chan, and B. Razavi. Stacked inductors and transformers in cmos technology. IEEE Journal of Solid-State Circuits, 36(4):620-628, Apr 2001.

[8] W. Y. Yin, J. Y. Xie, K. Kang, J. Shi, J. F. Mao, and X. W. Sun. Vertical topologies of miniature multispiral stacked inductors. IEEE Transactions on Microwave Theory and Techniques, 56(2):475-486, Feb 2008.

[9] G. Haobijam and R. Paily. Quality factor enhancement of cmos inductor with pyramidal winding of metal turns. In 2007 International Workshop on Physics of Semiconductor Devices, pages 729-732, Dec 2007.

[10] Chih-Chun Tang, Chia-Hsin Wu, and Shen-Iuan Liu. Miniature 3-d inductors in standard cmos process. IEEE Journal of Solid-State Circuits, 37(4):471-480, Apr 2002.

[11] A. Maric, G. Radosavljevic, M. Zivanov, L. Zivanov, G. Stojanovic, M. Mayer, A. Jachimowicz, and F. Keplinger. Modelling and characterisation of fractal based rf inductors on silicon substrate. In 2008 International Conference on Advanced Semiconductor Devices and Microsystems, pages 191-194, Oct 2008.

[12] G. Wang, L. Xu, and T. Wang. A novel mems fractal inductor based on hilbert curve. In 2012 Fourth International Conference on Computational Intelligence and Communication Networks, pages 241-244, Nov 2012.

[13] N. Lazarus, C. D. Meyer, and S. S. Bedair. Fractal inductors. IEEE Transactions on Magnetics, 50(4):1-8, April 2014.

[14] G. Shoute and D. W. Barlage. Fractal loop inductors. IEEE Transactions on Magnetics, 51(6):1-8, June 2015.

[15] D. K. Jair, M. C. Hsieh, C. S. Lin, S. M. Chen, and Y. H. Chen. The development of the high performance parallelstacked rf spiral inductor. In 2009 Symposium on Design, Test, Integration Packaging of MEMS/MOEMS, pages 424427, April 2009. 